\title{
Matrine inhibits BCR/ABL mediated ERK/MAPK pathway in human leukemia cells
}

\author{
Lingdi Ma ${ }^{1, *}$, Zhenyu $\mathrm{Xu}^{2, *}$, Jian Wang ${ }^{1}$, Zhichao Zhu ${ }^{3}$, Guibin Lin ${ }^{1}$, Lijia Jiang ${ }^{3}$, \\ Xuzhang Lu $^{4}$ and Chang Zou ${ }^{5}$ \\ 'Laboratory Center, The Third People's Hospital of Huizhou, Affiliated Hospital of Guangzhou Medical University, Huizhou \\ 516002, China \\ ${ }^{2}$ Department of Pharmacy, Yijishan Affiliated Hospital of Wannan Medical College, Wuhu 241001, China \\ ${ }^{3}$ Laboratory Center, The Second People's Hospital of Changzhou, Affiliated Hospital of Nanjing Medical University, Changzhou \\ 213000, China \\ ${ }^{4}$ Department of Hematology, The Second People's Hospital of Changzhou, Affiliated Hospital of Nanjing Medical University, \\ Changzhou 213000, China \\ ${ }^{5}$ Clinical Medical Research Center, The Second Clinical College of Jinan University, Shenzhen People's Hospital, Shenzhen \\ 518020, China \\ "These authors have contributed equally to this work
}

Correspondence to: Lingdi Ma, email: lingdimawsh@126.com

Keywords: matrine; ERK/MAPK; BCR/ABL; chronic myelogenous leukemia (CML); K562 cells

Received: August 20, $2016 \quad$ Accepted: June 30, $2017 \quad$ Published: November 10, 2017

Copyright: Ma et al. This is an open-access article distributed under the terms of the Creative Commons Attribution License 3.0 (CC BY 3.0), which permits unrestricted use, distribution, and reproduction in any medium, provided the original author and source are credited.

\section{ABSTRACT}

The BCR/ABL fusion gene and its downstream signaling pathways such as Ras/ Raf/MAPK, JAK/STAT3, and PI3K/AKT pathways play important roles in malignant transformation of leukemia, especially chronic myelogenous leukemia (CML). Our previous study showed that matrine, an alkaloid extracted from a Chinese herb radix sophorae, significantly inhibited the proliferation of human CML K562cells, induced cell cycle arrest in G0/G1, and promoted cell apoptosis. In the present study, we investigated the molecular mechanism of matrine in the growth inhibition of leukemia cells using K562 and HL-60 cell lines. RT-PCR and Western blot assay demonstrated that the expression of BCR/ABL in $\mathrm{K562}$ and HL-60 cells was significantly inhibited by matrine treatment. Phosphorylation of MEK1, ERK1/2, and their upstream adaptor molecules Shc and SHP2 were significantly downregulated. The protein and mRNA expression of components of the ERK/MAPK signal pathway, and Bcl-xL, Cyclin D1, and c-Myc, were dramatically reduced. Conversely, the expression of p27, a negative regulator of cell cycle progression, increased after matrine treatment. These results indicated that the inhibition of ERK/MAPK and BCR/ABL signaling pathway was associated with matrine's suppressive effects on the growth of $\mathrm{K562}$ and HL-60 cells. In in vivo study, matrine significantly decreased the mortality rate of tumor-baring mice and suggested that matrine could exert its anti-leukemia effect in vivo.

\section{INTRODUCTION}

Chronic myeloid leukemia (CML) is a hematopoietic stem cell malignancy characterized by a translocation between chromosomes 9 and 22 in which the translocated chromosome 22 is also known as the Philadelphia chromosome [1]. $\mathrm{BCR} / \mathrm{ABL}$ is required for the pathogenesis of CML by activating multiple intracellular signal transduction pathways such as Ras/Raf/MAPK, JAK/ STAT3, and PI3K/AKT via its protein tyrosine kinase (PTK) interfering with the development of hematopoietic stem cells or progenitor cells. Thus, targeting the signaling 
pathways activated by $\mathrm{BCR} / \mathrm{ABL}$ is a promising approach for drug development to treat CML [2-5]. Imatinib mesylate (IM), a small molecule inhibitor of the BCR/ABL kinase, is very effective in the treatment of CML, but the high risks of drug toxicity, drug resistance, and recurrence after drug withdrawal greatly limit its application in accelerated and blastic CML $[6,7]$. A better understanding of molecular mechanisms underlying the human hematopoietic progenitor transformation in CML is essential to developing alternative approaches to target leukemogenic cells in CML.

Among the downstream signaling pathways activated by $\mathrm{BCR} / \mathrm{ABL}$ tyrosine kinase, the abnormal activation of mitogen-activated protein kinase (MAPK) pathway plays an important role in the occurrence and development of CML $[8,9]$. MAPK is a member of a protein kinase family with highly conservative serine/ threonine protein kinase, which could be activated by a series of extracellular signaling molecules and stimulus such as growth factors and cytokines as well as osmotic pressure changes. MAPK is involved in the regulation of cell proliferation and differentiation. There are three branches of the MAPK cascade, including extracellular signal regulated kinase (ERK), c-Jun N-terminal kinase (JNK), and p38 MAP kinase, that are activated by different up-stream signals and exert independent effects on regulations of distinct physiological processes. These three branches can be activated by various cell stimuli, such as growth factors, cytokines, and cellular stress. ERK mainly responds to growth factors and promotes cell growth. JNK and p38 MAPK have multiple effects, depending on the stimulus and the environment in which activations occur. Among these pathways, ERK/MAPK pathway is considered to be a classic MAPK signaling cascade. The general scheme is as follows: epidermal growth factor (EGF) in plasma binds to its specific receptor on the cell membrane, leading to its dimerization as it binds to the Src homology-2 domain of the adapter protein, growth factor receptor bound protein2 (Grb2), on the cell membrane by self-phosphorylation. With the formation of Grb2-SOS complex by Grb2 and G protein exchange factor (SOS), the Ras-GDP protein is recruited to the cell membrane and promotes the dissociation of Ras from GDP, which then binds to GTP to induce the formation of an active RasGTP complex. The activated Ras sequentially activates the downstream serine/threonine protein kinase including Raf-1, MEK (a MAP kinase), and MAPKs (ERK, JNK and p38MAPK). It is involved in the regulation of cell survival, cell proliferation, cell differentiation, cell cycle progression, and induction of leukemogenesis [10-13].

Matrine is an alkaloid extracted from Chinese herb radix sophorae. It has potent anti-inflammatory, anti-oxidative, anti-infective and immune regulatory effects [14-16]. Studies revealed that matrine might exert inhibitory effects on the growth of many kinds of tumors such as gastric cancer, rhabdomyosarcoma, acute myeloid leukemia, and breast cancer $[17,18]$. In a previous study, we found that matrine effectively inhibited the proliferation of CML K562 cells and induced cell apoptosis. Further study indicated that matrine might inhibit the proliferation of leukemia cells via suppressing intracellular DNA synthesis, blocking mitosis, and inducing apoptosis [19]. Recent studies showed that matrine was able to inhibit the Ras/MAPK signaling pathway to exert anti-tumor effect on gastric cancer [20]. Although the anti-tumor effect of matrine has been studied in recent years, the mechanism underlying this effect is still poorly understood.

Here we investigated the potential molecular mechanism underlying the effects of matrine on CML K562 and HL-60 cells. Our results identified that antileukemic effects of matrine were correlated with the inhibition of ERK/MAPK signaling activation and decreased expression of upstream $\mathrm{BCR} / \mathrm{ABL}$, which suggested that matrine might be of therapeutic utility in patients with CML.

\section{RESULTS}

\section{Matrine inhibited Ras/Raf/ERK/MAPK signaling through down-regulating phosphorylation of MEK1 and ERK1/2}

Ras is an initial activator in Ras/Raf/ERK/MAPK signaling transduction pathway. Grb2 binds with SOS to become the Grb2-SOS complex, which activates Ras, and is then followed by activations of a MAP kinase kinase kinase (MKKK), activations of MAP kinase kinase (MKK) by MKKK, and finally activations of MAPK by MKK with phosphorylation $[12,13]$. The protein of Src homology 2 domain (SPH2) and Src homology 2 domain containing protein $\mathrm{C}$ (Shc) play important roles in Grb2 recruitment and Ras activation [21,22]. To assess whether the effect of matrine on leukemia cells is involved in the inactivation of Ras/Raf/ERK/MAPK signaling pathway, the expression of MEK, ERK1/2, phospho-MEK, phospho-ERK1/2 protein and the upstream adaptor molecules SHP2 and Shc were detected by Western blot. The ratio of phosphorylated protein to total protein was used to assess the influence of matrine on the protein phosphorylation. Our results showed that matrine treatment for $48 \mathrm{~h}$ could significantly reduce the expressions of phosphorylated MEK1, ERK1/2 in both K562 and HL-60 cells without changing the protein expressions of total MEK1, ERK1/2 (Figure 1A and 1B).

In the classic Ras/Raf/MEK/ERK signaling pathway, Shc is an upstream molecule in the activation of Ras/MAPK pathway. The phosphorylation of tyrosine residues of She may provide a binding site to Grb2 to become a Shc-Grb2 complex. The cytoplasmic Grb2-SOS complex is recruited to the cell membrane, which leads to the activation of Ras-mediated MAPK cascade [22]. SHP2 is a non-receptor protein tyrosine phosphatase containing two Src homology-2 domains. Almost all cytokinesor growth factors-mediated Ras/MAPK activation is 
dependent on the activation of SHP2. EGFR, Grb2, and SOS may serve as substrates of SHP2 and directly bind to SHP2, leading to Ras activation. In addition, SHP2 may block the hydrolysis of Ras-GDP by GTP, which could prolong the half-life of Ras-GTP, resulting in the continuous activation of Ras/MAPK signaling pathway $[21,23]$. It was also found in this study that matrine significantly reduced the expressions of phosphorylated Shc and SHP2 but failed to affect the expressions of total proteins of these molecules (Figure 1C and 1D). These results suggest that matrine might affect the cell growth and proliferation through regulating the activities of ERK MAPK signaling pathway in both K562 and HL-60cells.

\section{Matrine down-regulated Cyclin D1, c-Myc, and Bcl-xL and, up-regulated p27}

We investigated the influence of matrine on Cyclin D1, c-Myc, p27, and Bcl-xL as they are important molecular factors involved in cell cycle progression, apoptosis, and proliferation. The Western blot results showed that matrine significantly reduced the expressions of cell cycle regulators Cyclin D1, c-Myc, and antiapoptotic protein $\mathrm{Bcl}-\mathrm{xL}$. Under the same conditions, the negative regulator of cell cycle p27 protein was up-regulated in both K562 and HL-60 cells (Figure 2A and $2 \mathrm{~B})$. RT-PCR results confirmed the significantly attenuated expression of Bcl-xL, Cyclin D1, and c-Myc mRNA in both K562 and HL-60 cells in response to matrine treatment (Figure 2C and 2D). These findings were consistent with the cell proliferation inhibition and the induction of cell cycle arrest by matrine treatment in our previous study. Cyclin D1 plays a crucial role in the regulation of cell cycle progression, which could promote cells to cross the G1/S checkpoint and promote the cell cycle progression [24]. c-Myc, a product of oncogene c-Myc, may regulate the expressions of Cyclin D, E, and A, promote the cell cycle transition from $\mathrm{G} 1$ phase to $\mathrm{S}$ phase as well as regulate cell proliferation and apoptosis [25]. p27 is a negative regulator of cell cycle progression, which inhibits the formation of Cyclin D/CDK2 complex and block cells in G1 phase [26]. The above findings indicated that cell cycle blockage and apoptosis in K562 cells induced by matrine were related to the regulation of
A

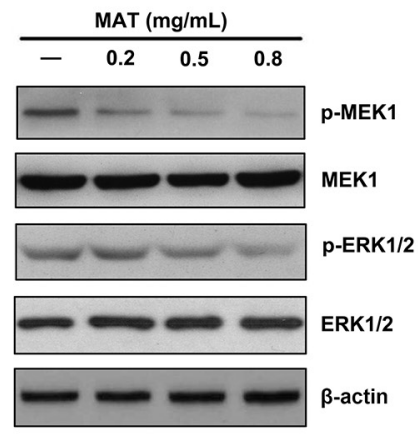

C
B

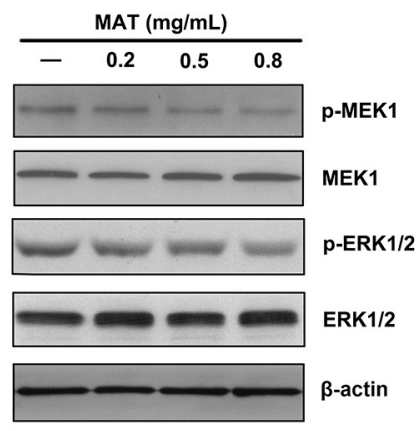

D

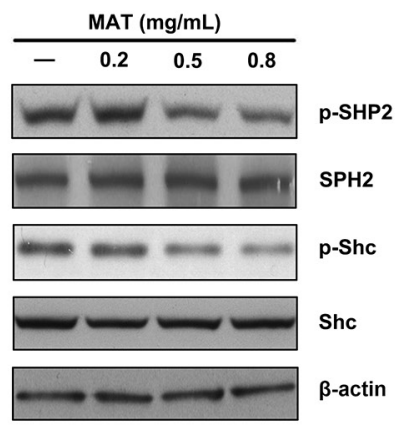

Figure 1: Matrine down-regulated the phosphorylation levels of effectors of ERK/MAPK signaling pathway in both K562 and HL-60 cells. Matrine inhibited the expressions of phosphorylated MEK1 and ERK1/2. After $0.2,0.5$ and $0.8 \mathrm{mg} / \mathrm{ml} \mathrm{matrine}$ treatment for 48h, the expressions of phospho-MEK and phospho-ERK1/2 were reduced significantly in K562 (A) and HL-60 cells (B); (C) \& (D): Matrine down-regulated the expressions of phospho-SHP2 and phospho-Shc (two up-stream adaptor molecules of ERK/MAPK pathway). After matrine treatment for $48 \mathrm{~h}$, the protein expressions of phospho-SHP2 and phospho-Shc were reduced significantly. 
Cyclin D1, c-Myc, p27, and Bcl-xL expressions. Since these molecular factors could be responsive to the Ras/ MAPK pathway [27-29], and the above results indicated that matrine significantly reduced phosphorylation levels of ERK, MEK, SHP2 as well as Shc, it suggested that matrine inhibited CML cell growth through the MEK/ MAPK pathway.

\section{BCR/ABL were involved in matrine-induced inhibition of ERK/MAPK pathway}

The oncogenic $\mathrm{BCR} / \mathrm{ABL}$ tyrosine kinase is essential for leukemogenesis. BCR/ABL-mediated signal transduction pathways could interfere with various cellular physiological processes, including cells proliferation,

A

B
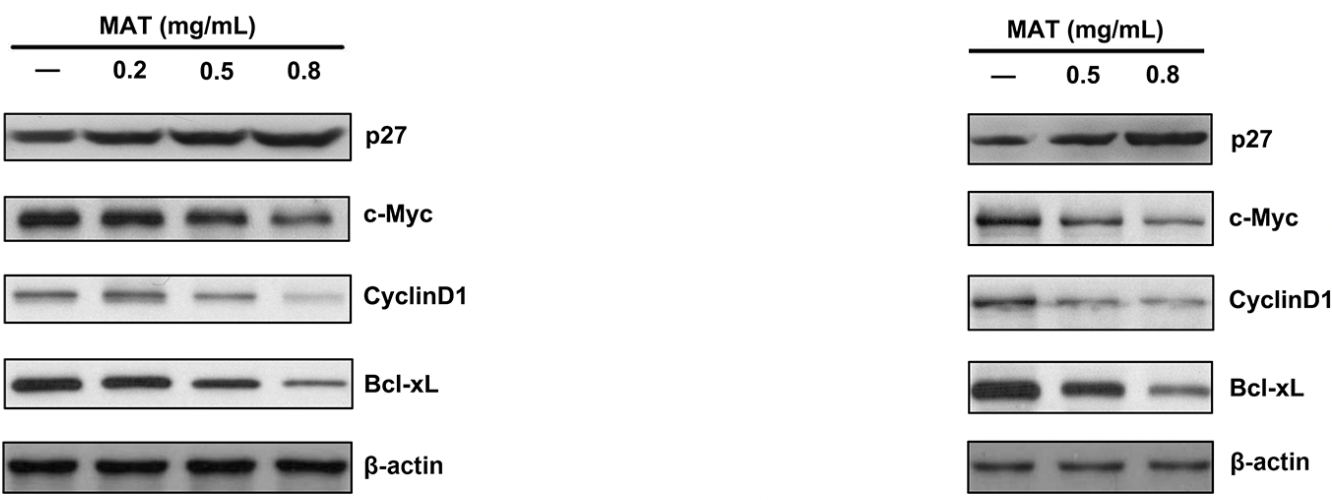

C
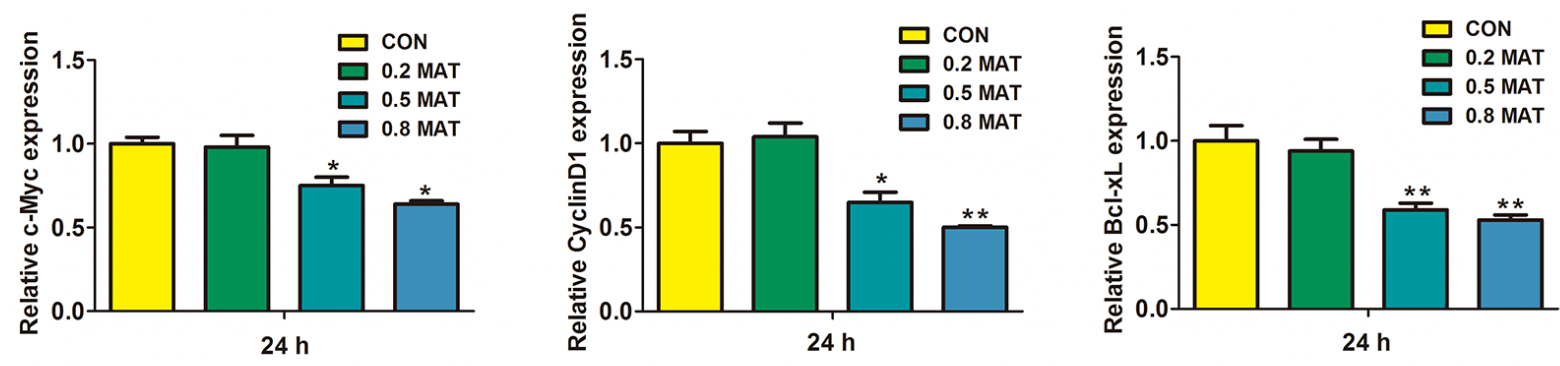

D
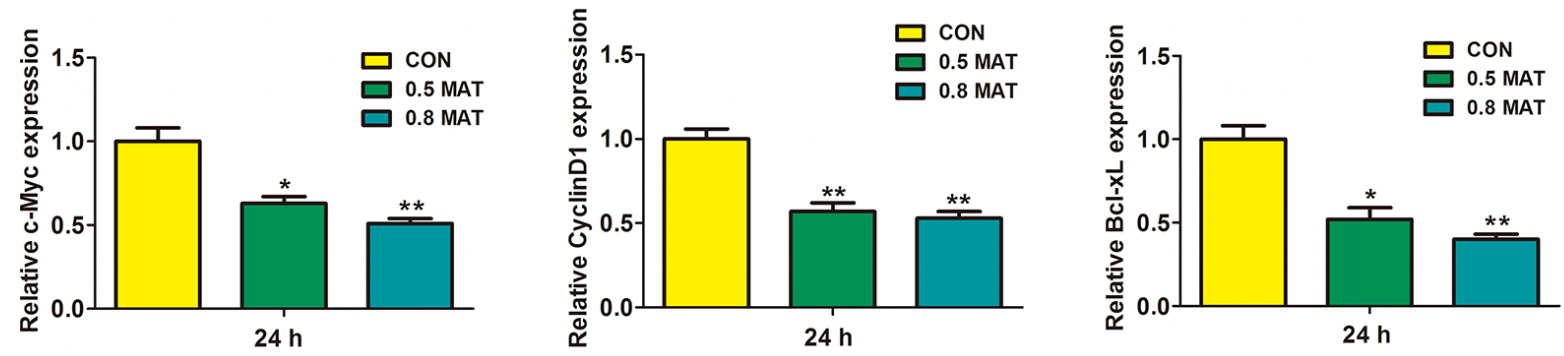

Figure 2: Effects of matrine on the expressions of molecules regulated by ERK/MAPK pathway. Results of Western blot assay showed that $48 \mathrm{~h}$ treatment with 0.5 and $0.8 \mathrm{mg} / \mathrm{ml}$ matrine significantly inhibited the expressions of c-Myc, Cyclin D1, and Bcl-xL proteins, but significantly increased p27 protein expression in K562 (A) and HL-60 cells (B). RT-PCR results showed that 48h matrine treatment inhibited the mRNA expressions of c-Myc, Cyclin D1, and Bcl-xL in a dose-dependent manner in K562 (C) and HL-60 cells (D). ${ }^{* *} \mathrm{p}<0.01$ vs. CON; ${ }^{\mathrm{p}}<0.05$ vs. CON. 
transformation, and apoptosis, which made the BCR/ ABL kinase an attractive target for clinical intervention in CML $[4,5]$. There were extensive biochemical evidences suggesting that BCR/ABL activated ERK through the activation of the RAS/RAF/ERK/MAPK pathway [30, 31], but the mechanism by which matrine regulates the activation of ERK/MAPK pathway and whether this mechanism involves BCR/ABL are still unclear. To assess whether the effect of matrine on ERK/MAPK is involved in the activation of BCR/ABL-MAPK axes, Western blot was used to evaluate the expression of $\mathrm{BCR} / \mathrm{ABL}$ fusion protein in both K562 and HL-60 cells. Results of this study demonstrated that matrine down-regulated BCR/ ABL protein expression (Figure $3 \mathrm{~A}$ and $3 \mathrm{~B}$ ) and mRNA levels of $\mathrm{BCR} / \mathrm{ABL}$ (Figure $3 \mathrm{C}$ and $3 \mathrm{D}$ ), indicating that anti-leukemic activities of matrine were related to $\mathrm{BCR} /$ ABL expression.

\section{Matrine down-regulated phosphorylated Src kinase}

Src protein, a type of non-receptor protein tyrosine kinases, is a member of Src family kinases (SFK). Several reports indicated that SFKs played an important role in the pathogenesis of $\mathrm{BCR} / \mathrm{ABL}+$ myelogenous leukemia $[32,33]$. To ascertain whether matrine plays a role in the modulation of Src activity, the expression of Src and phosphor-Src in both K562 and HL-60 cells after matrine

A
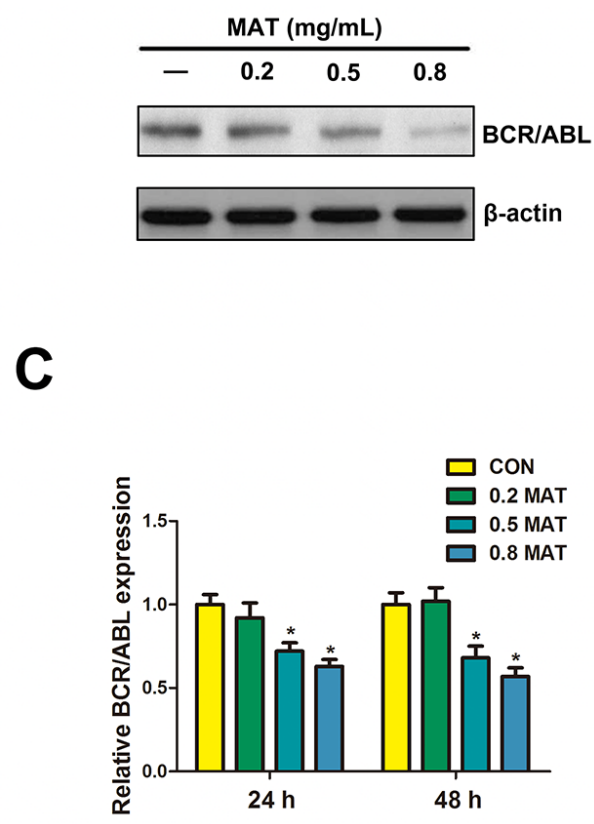

treatment were tested. The results showed that treatment with matrine for $48 \mathrm{~h}$ has no effects on the expression of total Src protein, but the expression of phosphor-Src was reduced significantly, suggesting that Src inhibition involved in the down regulating effects of matrine on the ERK/MAPK signaling pathway (Figure 4).

\section{Administration of matrine benefited total survival time of mice inoculated with human leukemia cells}

Previously, we reported that matrine could inhibit the cell growth of human leukemia cells K562. To further confirm its anti-leukemia effect in vivo, matrine(10, 20, $40 \mathrm{mg} / \mathrm{kg}$ ) as well as saline was injected in to mice with human leukemia cells very two days for 60 days. Survival rates were recorded and Kaplan-Meier survival curves showed that treatment of mice with matrine significantly decreased the mortality rate compared with mice that received saline in a dosage depend manner (Figure 5). These results indicated that matrine could exert its antileukemia effect in vivo.

\section{DISCUSSION}

In a previous study, it was found that matrine induced a concentration-and time-dependent decrease in the number of human CML K562 cells with increasing cell apoptosis

B

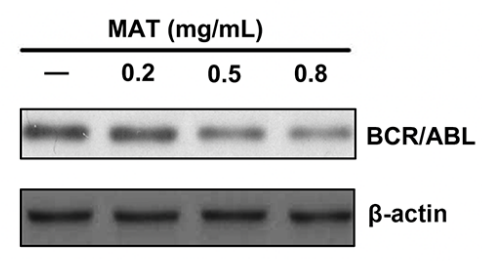

D

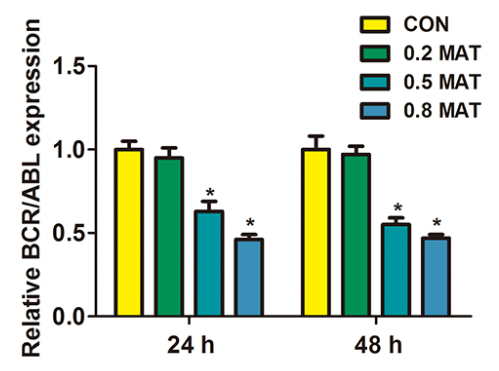

Figure 3: Matrine inhibited Bcr/Abl expression in K562 and HL-60 cells. After matrine treatment for $48 \mathrm{~h}$, the protein in K562 (A) and HL-60 cells (B), and mRNA expression in K562 (C) and HL-60 cells (D) levels of Bcr/Abl were reduced significantly when compared with the control group. ${ }^{* *} \mathrm{p}<0.01$ vs. CON; ${ }^{*} \mathrm{p}<0.05$ vs. CON. 
and significant $\mathrm{G} 0 / 1 \rightarrow \mathrm{S}$ cell cycle arrest, suggesting that matrine did not induce anti-proliferative effects through direct cytotoxicity [34], but through the blockage of cell cycle progression and induction of apoptosis. The aim of this study was to investigate whether matrine exerted

A

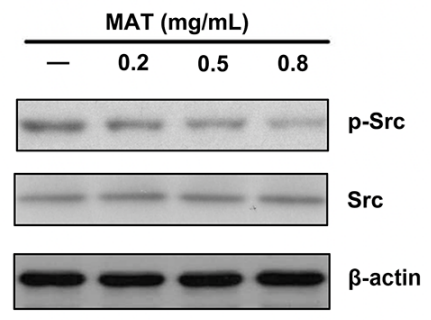

C

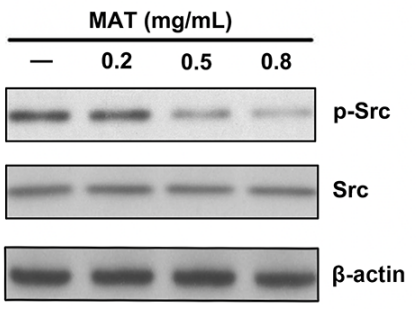

its anti-leukemic effects through inhibiting the ERK/ MAPK signaling pathway in both K562 and HL-60 cells. The cell cycle is mainly regulated in G1 phase, which determines the transition from G1 phase to $\mathrm{S}$ phase. Cyclin D1, c-Myc, and p27 are important proteins involved

B

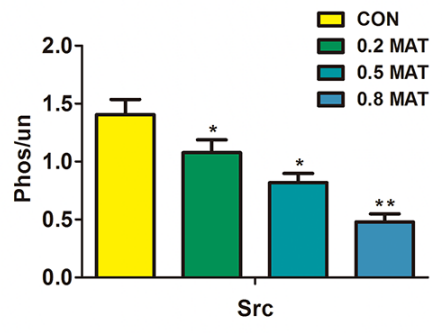

D

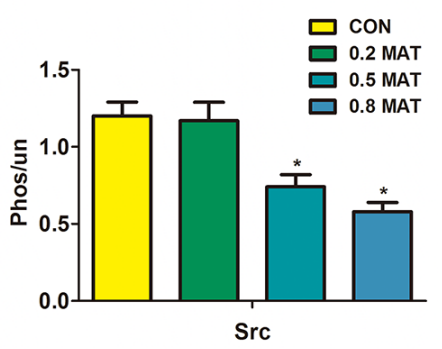

Figure 4: Matrine reduced the level of phosphorylated Src. After treatment with matrine for 48h, the expression of phospho-Src level was reduced significantly inK562 (A and B) and HL-60 cells (C and D). ${ }^{* *}$ p $<0.01$ vs. CON.

\section{Survival proportions: K562 models}

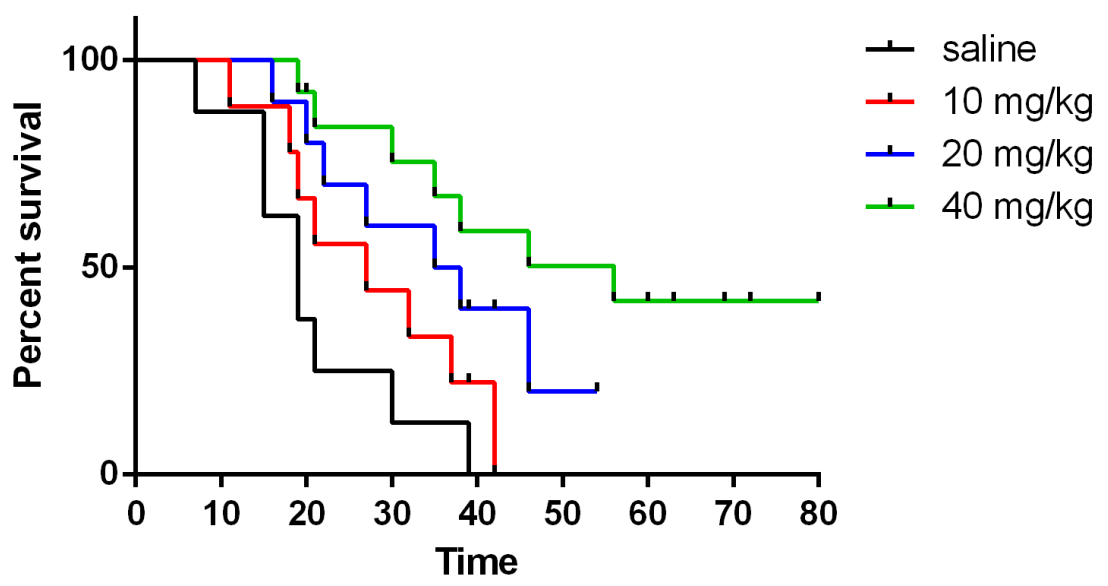

Figure 5: Matrine alleviates AML burden in vivo. Administration of Matrine could benefit the total survival of mice inoculated with K562. Median survival of $10 \mathrm{mg} / \mathrm{kg}$ group, $20 \mathrm{mg} / \mathrm{kg}$ group and $40 \mathrm{mg} / \mathrm{kg}$ group is days, 27 days and 36.5 days and 56 days separately, which are significant longer than the saline group (19 days, $\mathrm{P}<0.01$ ). 


\begin{tabular}{|c|c|c|}
\hline Gene & Sequences & Size (bp) \\
\hline \multirow{2}{*}{$\mathrm{BCR} / \mathrm{ABL}$} & 5’- CTCCAGACTGTCCACAGCATTCCG & \multirow{2}{*}{168 or 93} \\
\hline & 3'- CAGACCCTGAGGCTCAAAGTCAGA & \\
\hline \multirow{2}{*}{ Bcl-xL } & 5'- CCCAGAAAGGATACAGCTGG & \multirow{2}{*}{448} \\
\hline & 3'- GCGATCCGACTCACCAATAC & \\
\hline \multirow{2}{*}{ CyclinD1 } & 5'- CTGGCCATGAACTACCTGGA & \multirow{2}{*}{483} \\
\hline & 3'- GTCACACTTGATCACTCTGG & \\
\hline \multirow{2}{*}{ c-Myc } & 5'- CTCTCAACGACAGCAGCCCG & \multirow{2}{*}{250} \\
\hline & 3'- CCAGTCTCAGACCTAGTGGA & \\
\hline \multirow{2}{*}{ GAPDH } & 5'- ACCACAGTCCATGCCATCAC & \multirow{2}{*}{450} \\
\hline & 3'- TCCACCACCCTGTTGCTGTA & \\
\hline
\end{tabular}

in the regulation of G1 phase. Analyses of the regulatory molecules revealed that matrine-induced G1 block was associated with a significant down-regulation of the cyclin D1 and c-Myc expression and increased expression of p27 (a cyclin-dependent kinase inhibitor). Since these molecules responds to the ERK/MAPK signaling pathway, the effects of matrine on ERK/MAPK pathway was then investigated. As expected, matrine treatment resulted in significantly reduced levels of phosphorylated MEK and ERK, the key signaling molecules of the Ras/Raf/ERK/ MAPK oncogenic signaling pathway, thus confirming the potential suppressive effects of matrine on ERK/MAPK signaling. All these data suggested that anti-leukemic effects of matrine are mediated, at least in part, by inhibition of aberrantly activated ERK/MAPK signaling in both K562 and HL-60 cells.

The anti-apoptotic activity of BCR/ABL contributes greatly to the development of CML. BCR/ABL may function either by enhancing the proliferation potential of hematopoietic progenitors or by preventing these progenitor cells from apoptosis. These processes are thought to be involved in intracellular signaling pathways, such as ERK/MAPK, JAK/STAT3, and PI3K/AKT pathways. As a driving force for CML, the activated tyrosine kinase of $\mathrm{BCR} / \mathrm{ABL}$ is able to stimulate the MAPK/ERK pathway. Studies have shown that after phosphorylation at Tyr177, BCR/ABL might bind to the Sre homology-2 domain of Grb2 to create the BCR/ABL/Grb2 complex, which then recruit cytoplasmic SOS and switched into the BCR/ABL/ Grb2/SOS complex, resulting in the activation of GDP-Ras $[8,35]$. The activation of Ras resulted in activation of its downstream effectors such as Raf-1, MEK, and MAPK, and was responsible for the activation of ERK/MAPK pathway to promote myeloid and lymphoid transformation. Results showed that matrine treatment significantly inhibited the mRNA and protein expressions of BCR/ABL in dose- and time-dependent manners, suggesting that inhibition of BCR/
ABL by matrine contributed to the growth inhibitions and the interruptions of ERK/MAPK signal network in both K562 and HL-60 cells.

Although $\mathrm{BCR} / \mathrm{ABL}$ is considered the trigger of malignant transformation in CML, targeting $\mathrm{BCR} /$ ABL kinase activity alone may not be sufficient for the leukemogenesis of $\mathrm{CML}$, for downstream pathways of $\mathrm{BCR} / \mathrm{ABL}$ can be activated independently of $\mathrm{BCR} / \mathrm{ABL}$ [36]. Reduction in phosphorylation levels could be the results of inhibition of other protein tyrosine kinases or activation of protein tyrosine phosphatase. The inhibitory effects of matrine on the phosphor tyrosine levels of ERK and MEK may not be precisely regulated by the suppression of upstream protein tyrosine kinases BCR/ ABL. There was evidence of an important role of SFKs in CML progression. The SFKs intracellular non-receptor tyrosine kinases are collaborative oncogenic kinases in $\mathrm{BCR} / \mathrm{ABL}$-induced leukemia and may act to couple BCR/ $\mathrm{ABL}$ to certain downstream signaling pathways involved in leukemic transformation. The activity of BCR/ABL can be enhanced through SFK-mediated phosphorylation within the activation loop of ABL, which was found to increase the $\mathrm{ABL}$ kinase activity in the progression of $\mathrm{CML}$ and $\mathrm{Ph}+$ ALL. In BCR/ABL+CML cells, activated Src may induce the phosphorylation of BCR/ABL at Tyr89 and Tyr245 and further increase the tyrosine kinase activity of $\mathrm{BCR} / \mathrm{ABL}$ [37]. Increasing preclinical and clinical evidence implicated that SFKs are also involved in imatinib resistance, which may be a cause of resistance to imatinib through BCR/ $\mathrm{ABL}$ independently in $\mathrm{Ph}+$ leukemia $[38,39]$. SFKs are also related to the Ras-mediated activation of ERK/MAPK pathway. SHP2 could activate Src to phosphorylate upstream adapter molecules, such as Shc, Grb2, and Gab2, leading to Ras/MAPK activation [40, 41]. In this study, we found that treatment with matrine had no influence on total Src protein expression, but reduced the phosphorylated Src significantly, suggesting that Src inhibition could be 
involved in the effects of matrineonbothK 562 and HL-60 leukemic cells and that matrine may be a dual inhibitor for SFK and BCR/ABL.

In conclusion, we have documented that the underlying mechanisms of matrine's anti-CML activity were involved in the inhibition of MAPK/ERK signaling pathway and down-regulated expression of BCR/ABL. Matrine is also found to inhibit the activation of Src kinase, suggesting that matrine may be an effective inhibitor of both SFK and MAPK/ERK. Further investigation of matrine on the MAPK/ERK-BCR/ABL-related signaling pathways would elucidate its molecular mechanisms underlying the anti-leukemic effects and its potential in the clinical therapy of CML and Ph+ ALL.

\section{MATERIALS AND METHODS}

\section{Reagents and antibodies}

Matrine with purity up to $99.5 \%$ was purchased from the Institute of Phytochemistry of Xi'an Botanical Garden in Shan'Xi Provincial Academy of Sciences. A stock solution $(10 \mathrm{mg} / \mathrm{ml})$ was prepared by dissolving matrine in ddH2O and stored at $-20^{\circ}$ C.K562 and HL-60cells were obtained from Shanghai Cell Bank of Chinese Academy of Science,Shanghai, China, and maintained in RPMI 1640 (Gibco, USA) containing 10\% fetal bovine serum (Gibco, USA) in an environment with $5 \% \mathrm{CO} 2$ at $37^{\circ} \mathrm{C}$. Primers for BCR/ABL, Bcl-xL, CyclinD1, and c-Myc were designed by Shanghai Sangon Co., Ltd. Antibodies against BCR/ABL (3908s), Bcl-xL (2762), CyclinD (2922), c-Myc (9402), p27 (2552) were purchased from Cell Signaling Technology (Beverly, MA, USA). Monoclonal antibodies against MEK1(YT2711), phosphoMEK1 (Ser217)(YP0167), ERK1/2(YT1625), phosphoERK1/2 (Tyr204)(YP0101), SHP2(YT4295), phosphorSHP2(Tyr580)(YP0582), Shc(YT4287), phospho-Shc (Tyr349) (YP0578) were purchased from Immunoway Biotechnology (Immunoway, USA). Src (anti-4878) and phospho-Src (Tyr418) were from Abnova, Taiwan.

\section{Cell culture}

K562 or HL-60 cells in logarithmic growth phase at $0.5-1.0 \times 10^{5} / \mathrm{ml}$ were treated with matrine at $0.2 \mathrm{mg} / \mathrm{ml}$, $0.5 \mathrm{mg} / \mathrm{ml}$ and $0.8 \mathrm{mg} / \mathrm{ml}$. In the control group, PBS of an equal volume was added. Cells were maintained in a humidified environment with $5 \% \mathrm{CO} 2$ at $37^{\circ} \mathrm{C}$ for $24 \mathrm{~h}$ and $48 \mathrm{~h}$. Cells were collected, and total RNA and protein were extracted for RT-PCR and Western blot assay.

\section{Real-time reverse transcription-PCR}

Real-time reverse transcription-PCR for estimation of BCR/ABL, Bcl-xL,CyclinD1, and c-Myc mRNAs were carried out by using the following primers (Table 1). Total
RNA was extracted from matrine-treated and untreated K562 or HL-60 by using TRIzol reagent (Gibco, USA). Five micrograms of RNA was reversely transcribed into cDNA using Revert Aid ${ }^{\mathrm{TM}}$ First Strand cDNA Synthesis Kit (K1622; Fermentas Inc, USA). Transcribed cDNA was amplified and quantified by the real-time fluorescent quantitative PCR with a SYBR Green qPCR kit (TaKaRa). The relative expressions of every gene were assessed in comparison with the housekeeping gene glyceraldehyde-3phosphate dehydrogenase (GAPDH). Blank controls, that did not contain cDNA, were run in parallel. All samples were run in triplicate. Relative expression values with SE and statistical comparison (unpaired two-tailed t test) were obtained by using the Qgene software.

\section{Western blot}

K562 or HL-60 cells were treated with matrine for $48 \mathrm{~h}$, and cells were harvested and lysed by using RIPA lyses buffer (Beijing Biotech Co., Ltd) for the extraction of total protein. Protein concentration was quantified with BCA Protein Assay Kit (Merck Chemicals Shanghai Company). For the detection of target proteins and phosphorylated proteins, proper proteins were subjected to $12.5 \%$ SDS-PAGE at a constant of $30 \mathrm{~mA}$ and then transferred onto PVDF membrane at $4^{\circ} \mathrm{C}$ overnight. The membrane was blocked in 5\% non-fat milk and then probed with various primary antibodies (1:500 or 1:2000) at $4^{\circ} \mathrm{C}$ overnight. $\beta$-actin was used as an internal reference. The membrane was washed with TBST three times and then incubated with HRP-conjugated secondary antibody (1:5000) at $37^{\circ} \mathrm{C}$ for $4 \mathrm{~h}$. Visualization was performed using the enhanced chemiluminescence detection reagents (Amersham Pharmacia Biotech). Film was exposed under $\mathrm{X}$-ray. Representative images were captured.

\section{In vivo study}

K562 cells $\left(1^{*} 10^{7} /\right.$ mouse) were injected once via tail vein of NoD/SCID mice at 5-6 weeks old. 20 days after the tail vein injection, mice were divided into 4 groups randomly. Different dosages of matrine as well as saline were injected into each group by i.p. injection every two days accordingly. Total survival dates of each group were documented for two months and mice were sacrificed when they show dying signs.

\section{Statistical analysis}

Data are presented as mean \pm standard deviation $(\bar{x} \pm \mathrm{SD})$. One-way ANOVA was used for comparisons among groups and q test with Newman-keuls method for paired comparisons. $\mathrm{P}$ value less than 0.05 was considered statistically significant and was derived from 2-tailed statistical test. All statistical analyses were performed using the software SPSS 14.0. 


\section{CONFLICTS OF INTEREST}

None declared.

\section{GRANT SUPPORT}

This work was supported by the National Nature Science Foundation of China (81673644), Medical Scientific Research Foundation of Guangdong Province (A2016252), Huizhou City Science and Technology Bureau (2016Y151), and the University Natural Science Research Project of Anhui Province (KJ2016A732).

\section{REFERENCES}

1. McGahon A, Bissonnette R, Schmitt M, Cotter KM, Green DR, Cotter TG. BCR-ABL maintains resistance of chronic myelogenous leukemia cells to apoptotic cell death. Blood. 1994; 83:1179-1187.

2. Lugo TG, Pendergast AM, Muller AJ, Witte ON. Tyrosine kinase activity and transformation potency of bcr-abl oncogene products. Science (New York, NY). 1990; 247:1079-1082

3. Deininger MW, Goldman JM, Melo JV. The molecular biology of chronic myeloid leukemia. Blood. 2000; 96:3343-3356.

4. Ren R. Mechanisms of BCR-ABL in the pathogenesis of chronic myelogenous leukaemia. Nature Reviews Cancer. 2005; 5:172-183.

5. Brehme M, Hantschel O, Colinge J, Kaupe I, Planyavsky M, Kocher T, Mechtler K, Bennett KL, Superti-Furga G. Charting the molecular network of the drug target Bcr-Abl. Proceedings of the National Academy of Sciences of the United States of America. 2009; 106:7414-7419.

6. Hentschel J, Rubio I, Eberhart M, Hipler C, Schiefner J, Schubert K, Loncarevic IF, Wittig U, Baniahmad A, von Eggeling F. BCR-ABL- and Ras-independent activation of Raf as a novel mechanism of Imatinib resistance in CML. International Journal of Oncology. 2011; 39:585-591

7. Druker BJ, Guilhot F, O'Brien SG, Gathmann I, Kantarjian H, Gattermann N, Deininger MW, Silver RT, Goldman JM, Stone RM, Cervantes F, Hochhaus A, Powell BL, et al. Five-year follow-up of patients receiving imatinib for chronic myeloid leukemia. The New England Journal of Medicine. 2006; 355:2408-2417.

8. Puil L, Liu J, Gish G, Mbamalu G, Bowtell D, Pelicci PG, Arlinghaus R, Pawson T. Bcr-Abl oncoproteins bind directly to activators of the Ras signalling pathway. The EMBO Journal. 1994; 13:764-773.

9. Yu C, Krystal G, Varticovksi L, McKinstry R, Rahmani M, Dent P, Grant S. Pharmacologic mitogen-activated protein/ extracellular signal-regulated kinase kinase/mitogenactivated protein kinase inhibitors interact synergistically with STI571 to induce apoptosis in Bcr/Abl-expressing human leukemia cells. Cancer Research. 2002; 62:188-199.

10. Sebolt-Leopold JS, Herrera R. Targeting the mitogenactivated protein kinase cascade to treat cancer. Nature Reviews Cancer. 2004; 4:937-947.

11. $\mathrm{Wu}$ GS. Role of mitogen-activated protein kinase phosphatases (MKPs) in cancer. Cancer Metastasis Reviews. 2007; 26:579-585.

12. Porter AC, Vaillancourt RR. Tyrosine kinase receptoractivated signal transduction pathways which lead to oncogenesis. Oncogene. 1998; 17:1343-1352.

13. Niault TS, Baccarini M. Targets of Raf in tumorigenesis. Carcinogenesis. 2010; 31:1165-1174.

14. Fu S, Sun C, Tao X, Ren Y. Anti-in fl ammatory effects of active constituents extracted from Chinese medicinal herbs against Propionibacterium acnes. Natural Product Research. 2012; 26:1746-1749.

15. Wan XY, Luo M, Li XD, He P. Hepatoprotective and antihepatocarcinogenic effects of glycyrrhizin and matrine. Chemico-biological Interactions. 2009; 181:15-19.

16. Yang Y, Xiu J, Zhang X, Zhang L, Yan K, Qin C, Liu J. Antiviral effect of matrine against human enterovirus 71 . Molecules (Basel, Switzerland). 2012; 17:10370-10376.

17. Liu Y, Xu Y, Ji W, Li X, Sun B, Gao Q, Su C. Anti-tumor activities of matrine and oxymatrine: literature review. Tumour Biology. 2014; 35:5111-5119.

18. Sun M, Cao H, Sun L, Dong S, Bian Y, Han J, Zhang L, Ren S, Hu Y, Liu C, Xu L, Liu P. Antitumor activities of kushen: literature review. Evidence-based Complementary and Alternative Medicine. 2012; 2012:373219.

19. Liu XS, Jiang J. Molecular mechanism of matrine-induced apoptosis in leukemia K562 cells. The American Journal of Chinese Medicine. 2006; 34:1095-1103.

20. Luo C, Zhong HJ, Zhu LM, Wu XG, Ying JE, Wang XH, $\mathrm{Lu} \mathrm{WX}, \mathrm{Xu} \mathrm{Q}$, Zhu YL, Huang J. Inhibition of matrine against gastric cancer cell line MNK45 growth and its anti-tumor mechanism. Molecular Biology Reports. 2012; 39:5459-5464.

21. Cunnick JM, Dorsey JF, Munoz-Antonia T, Mei L, Wu J. Requirement of SHP2 binding to Grb2-associated binder-1 for mitogen-activated protein kinase activation in response to lysophosphatidic acid and epidermal growth factor. The Journal of Biological Chemistry. 2000; 275:13842-13848.

22. Bromann PA, Korkaya H, Courtneidge SA. The interplay between Src family kinases and receptor tyrosine kinases. Oncogene. 2004; 23:7957-7968

23. Dance M, Montagner A, Salles JP, Yart A, Raynal P. The molecular functions of Shp2 in the Ras/Mitogen-activated protein kinase (ERK1/2) pathway. Cellular Signalling. 2008; 20:453-459.

24. Casimiro MC, Velasco-Velazquez M, Aguirre-Alvarado C, Pestell RG. Overview of cyclins D1 function in cancer 
and the CDK inhibitor landscape: past and present. Expert Opinion on Investigational Drugs. 2014; 23:295-304.

25. Henriksson M, Luscher B. Proteins of the Myc network: essential regulators of cell growth and differentiation. Advances in Cancer Research. 1996; 68:109-182.

26. Toyoshima H, Hunter T. p27, a novel inhibitor of G1 cyclinCdk protein kinase activity, is related to p21. Cell. 1994; 78:67-74.

27. Terada Y, Inoshita S, Nakashima O, Kuwahara M, Sasaki S, Marumo F. Regulation of cyclin D1 expression and cell cycle progression by mitogen-activated protein kinase cascade. Kidney International. 1999; 56:1258-1261.

28. Hester KD, Verhelle D, Escoubet-Lozach L, Luna R, Rose DW, Glass CK. Differential repression of c-myc and cdc2 gene expression by ERF and PE-1/METS. Cell Cycle (Georgetown, Tex). 2007; 6:1594-1604.

29. Vitagliano O, Addeo R, D'Angelo V, Indolfi C, Indolfi P, Casale F. The Bcl-2/Bax and Ras/Raf/MEK/ERK signaling pathways: implications in pediatric leukemia pathogenesis and new prospects for therapeutic approaches. Expert Review of Hematology. 2013; 6:587-597.

30. Modi H, Li L, Chu S, Rossi J, Yee JK, Bhatia R. Inhibition of Grb2 expression demonstrates an important role in BCR-ABL-mediated MAPK activation and transformation of primary human hematopoietic cells. Leukemia. 2011; 25:305-312.

31. Chu S, Li L, Singh H, Bhatia R. BCR-tyrosine 177 plays an essential role in Ras and Akt activation and in human hematopoietic progenitor transformation in chronic myelogenous leukemia. Cancer Research. 2007; 67:7045-7053.

32. Li S. Src-family kinases in the development and therapy of Philadelphia chromosome-positive chronic myeloid leukemia and acute lymphoblastic leukemia. Leukemia \& Lymphoma. 2008; 49:19-26.

33. Roginskaya V, Zuo S, Caudell E, Nambudiri G, Kraker AJ, Corey SJ. Therapeutic targeting of Src-kinase Lyn in myeloid leukemic cell growth. Leukemia. 1999; 13:855-861.
34. Guo L, Xue TY, Xu W, Gao JZ. Matrine promotes G0/G1 arrest and down-regulates cyclin D1 expression in human rhabdomyosarcoma cells. Panminerva Medica. 2013; 55:291-296.

35. Zhang X, Subrahmanyam R, Wong R, Gross AW, Ren R. The NH(2)-terminal coiled-coil domain and tyrosine 177 play important roles in induction of a myeloproliferative disease in mice by Bcr-Abl. Molecular and Cellular Biology. 2001; 21:840-853.

36. Hamilton A, Helgason GV, Schemionek M, Zhang B, Myssina S, Allan EK, Nicolini FE, Muller-Tidow C, Bhatia R, Brunton VG, Koschmieder S, Holyoake TL. Chronic myeloid leukemia stem cells are not dependent on Bcr-Abl kinase activity for their survival. Blood. 2012; 119:1501-1510.

37. Cilloni D, Saglio G. Molecular pathways: BCR-ABL. Clinical Cancer Research. 2012; 18:930-937.

38. Donato NJ, Wu JY, Stapley J, Lin H, Arlinghaus R, Aggarwal BB, Shishodia S, Albitar M, Hayes K, Kantarjian $\mathrm{H}$, Talpaz M. Imatinib mesylate resistance through BCR$\mathrm{ABL}$ independence in chronic myelogenous leukemia. Cancer Research. 2004; 64:672-677.

39. Donato NJ, Wu JY, Stapley J, Gallick G, Lin H, Arlinghaus R, Talpaz M. BCR-ABL independence and LYN kinase overexpression in chronic myelogenous leukemia cells selected for resistance to STI571. Blood. 2003; 101:690-698.

40. Kim LC, Song L, Haura EB. Src kinases as therapeutic targets for cancer. Nature Reviews Clinical Oncology. 2009; 6:587-595.

41. Gotoh N, Toyoda M, Shibuya M. Tyrosine phosphorylation sites at amino acids 239 and 240 of Shc are involved in epidermal growth factor-induced mitogenic signaling that is distinct from Ras/mitogen-activated protein kinase activation. Molecular and Cellular Biology. 1997; 17:1824-1831. 